\title{
Fuzzy Function: Theoretical and Practical Point of View*
}

\author{
Irina Perfilieva,
}

University of Ostrava, Inst. for Research and Applications of Fuzzy Modeling, Irina.Perfilieva@osu.cz

\begin{abstract}
The aim of this investigation is to reconsider two notions of fuzzy function, namely: a fuzzy function as a special fuzzy relation and a fuzzy function as a mapping between fuzzy spaces. We propose to combine both notions in such a way that a fuzzy function as a relation determines a fuzzy function as a mapping. We investigate conditions which guarantee that dependent values of the related fuzzy functions coincide. Moreover, we investigate properties and relationship of the related fuzzy functions in the case when both of them are "fuzzified" versions of the same ordinary function.
\end{abstract}

Keywords: Fuzzy function, extensional function, fuzzy equivalence, extension principle

\section{Introduction}

The notion of fuzzy function has at least two different meanings in fuzzy literature. From theoretical point of view (see e.g., [1-4]), a fuzzy function is a special fuzzy relation with a generalized property of uniqueness. According to this approach, each element from a range of a fuzzy function can be assigned with a certain degree to each element from its domain. Thus, instead of working with direct functional values we have to work with degrees.

Another, practical point of view on a fuzzy function originates from the early work of L. Zadeh [5] where he proposed the well known extension principle. According to this principle, every function (in an ordinary sense) can be "fuzzified", i.e. extended to arguments given by fuzzy sets. Thus, any ordinary function determines a mapping from a set of fuzzy subsets of its domain to a set of fuzzy subsets of its range. In [6], we have used this approach and defined a fuzzy function as an ordinary mapping between two universes of fuzzy sets. Similar definition appeared in [7] and implicitly, in many other papers devoted to fuzzy IF-THEN rules models where these models are used as partially given fuzzy functions.

The aim of this investigation is to reconsider both notions and combine them in such a way that a fuzzy function as a relation determines a fuzzy function as a mapping (we will say that they are related). We will investigate conditions which guarantee that dependent values of related fuzzy functions

*The author was partially supported by the project MSM 6198898701 of the MŠMT ČR. coincide. Moreover, we will investigate properties and relationship of related fuzzy functions in the case when they are "fuzzified" versions of the same ordinary function.

The paper is organized as follows. In Sections 2, we give preliminary information about extension principle, residuated lattices, fuzzy sets and fuzzy spaces. Fuzzy functions and two approaches to this notion are discussed in Sections 3. Main results are proved in Section 4.

\section{Preliminaries}

\subsection{Extension principle and its relation form}

An extension principle has been proposed by L. Zadeh [5] in 1975 and since then it is widely used in the fuzzy set theory and its applications. Let us recall the principle and propose its relation form which will be later on used in a relationship to fuzzy function.

Assume that $X, Y$ are universal sets and $f$ : $X \longrightarrow Y$ is a function. Let moreover, $\mathcal{F}(X), \mathcal{F}(Y)$ be respective universes of fuzzy sets, identified with their membership functions, i.e. $\mathcal{F}(X)=\{A$ : $X \longrightarrow[0,1]\}$ and similarly, $\mathcal{F}(Y)$. By the extension principle, $f$ induces a function $\hat{f}: \mathcal{F}(X) \longrightarrow \mathcal{F}(Y)$ such that for all $A \in \mathcal{F}(X)$,

$$
\hat{f}(A)(y)=\sup _{y=f(x)} A(x) .
$$

Let $R_{f}$ be a binary relation on $X \times Y$ which corresponds to the function $f$, i.e.

$$
R_{f}(x, y)=1 \Leftrightarrow y=f(x) .
$$

Then it is easy to see that (1) can be equivalently represented by

$$
\hat{f}(A)(y)=\sup _{x \in X} \min \left(A(x), R_{f}(x, y)\right) .
$$

Expression (2) is the relation form of the extension principle. The meaning of expression (2) will be more general if fuzzy set $A$ is $L$-valued (see Definition 2 below), binary relation $R_{f}$ is a fuzzy relation, and min is replaced by a monoidal operation (see Subsection 2.2). In Section 3, we will discuss the proposed generalization and its relationship to fuzzy functions. 


\subsection{Residuated lattice}

Our basic algebra of operations is a residuated lattice*) $^{*}$

\section{Definition 1}

$A$ residuated lattice is an algebra

$$
\mathcal{L}=\langle L, \vee, \wedge, *, \rightarrow, \mathbf{0}, \mathbf{1}\rangle .
$$

with the support $L$, four binary operations and two constants such that

- $\langle L, \vee, \wedge, \mathbf{0}, \mathbf{1}\rangle$ is a lattice where the ordering $\leq$ is defined using operations $\vee, \wedge$, and $\mathbf{0 , 1}$ are the least and the greatest elements, respectively;

- $\langle L, *, \mathbf{1}\rangle$ is a commutative monoid where $*$ is a commutative and associative operation with the identity $a * \mathbf{1}=a$;

- the operation $\rightarrow$ is a residuation with respect to $*$, i.e.

$$
a * b \leq c \quad \text { iff } \quad a \leq b \rightarrow c .
$$

A residuated lattice is complete if it is complete as a lattice.

The following is an additional (derived) binary operation of $\mathcal{L}$ :

$$
x \leftrightarrow y=(x \rightarrow y) \wedge(y \rightarrow x) .
$$

The well known examples of residuated lattices are: boolean algebra, Gödel, Łukasiewicz and product algebras. In the particular case $L=[0,1]$, multiplication $*$ is a left continuous $t$-norm.

From now on we will fix a complete residuated lattice $\mathcal{L}$

\section{3. $L$-valued fuzzy sets, fuzzy relations and fuzzy spaces}

\section{Definition 2}

Let $X$ be a non-empty universal set, $\mathcal{L}$ a complete residuated lattice. An $L$-valued fuzzy set $A$ of $X$ (fuzzy set, shortly) is a map $A: X \longrightarrow L$ which is known as a membership function of $A$ as well. (Thus, a fuzzy set is identified with its membership function.) Fuzzy set $A$ is normal if there exists $x_{A} \in X$ such that $A\left(x_{A}\right)=1$. The (ordinary) set $\operatorname{Ker}(A)=\{x \in X \mid A(x)=1\}$ is a kernel of a normal fuzzy set $A$. The (ordinary) set $\operatorname{Supp}(A)=\{x \in X \mid A(x)>0\}$ is a support set of a fuzzy set $A$.

$A$ class of $L$-valued fuzzy sets of $X$ will be denoted $L^{X}$. A couple $\left(L^{X},=\right)$ is called a fuzzy space on $X$.

In the similar way we define a (binary) (L-valued) fuzzy relation as a fuzzy set of $X \times Y$. A fuzzy set of $X \times X$ is called a (binary) fuzzy relation on $X$.

Binary fuzzy relation $E$ on $X$ is called a similarity or a fuzzy equivalence on $X$ (see [8-10]) if for all $x, y, z \in X$, the following holds:

\footnotetext{
${ }^{*)}$ We assume that residuated lattice is a lattice-ordered commutative, residuated and integral monoid.
}

S.1 $E(x, x)=1$,

reflexivity,

S.2 $E(x, y)=E(y, x)$

S.3 $E(x, y) * E(y, z) \leq E(x, z)$

symmetry,

transitivity.

If a fuzzy equivalence $E$ fulfils

S.4 $E(x, y)=1$ if and only if $x=y$,

then it is called separated or a fuzzy equality on $X$.

A couple $(X, E)$, where $E$ is a fuzzy equivalence on $X$, is called an E-space of $X$. Below, we will show how fuzzy equivalence $E$ on $X$ helps to determine another space - a space of $E$-fuzzy points of $X$.

Given fuzzy equivalence $E$ on $X$, certain fuzzy sets of $X$ can be characterized as equivalence classes of single elements of $X$, i.e. as fuzzy sets determined by membership functions of the form $E_{t}, t \in X$, where $E_{t}(x)=E(t, x), x \in X$. Fuzzy set $E_{t}$ is called an $E$-fuzzy point of $X$ (determined by $t$ ). It is easy to see that $E_{t}$ is a normal fuzzy set and that $E_{t}$ can be determined by any element of its kernel. Denote

$$
X / E=\left\{E_{t} \mid t \in X\right\}
$$

a class of all $E$-fuzzy points of $X$. If we now consider $X / E$ as a new universal set then it can be proved that the following fuzzy relation

$$
\left(E_{t} \equiv E_{u}\right)=\bigwedge_{x \in X}\left(E_{t}(x) \leftrightarrow E_{u}(x)\right)
$$

is a fuzzy equality on $X /{ }_{E}$. Moreover (see [11]), for $t, u \in X$,

$$
\left(E_{t} \equiv E_{u}\right)=E(t, u) .
$$

Fuzzy equality $\left(E_{t} \equiv E_{u}\right)$ is a degree of coincidence between two $E$-fuzzy points $E_{t}$ and $E_{u}$. We will write $E_{t}=E_{u}$ if $\left(E_{t} \equiv E_{u}\right)=1$. By (4), $E_{t}=E_{u}$ if and only if $E(t, u)=1$, or if and only if $u$ belongs to the kernel of $E_{t}$.

The couple $\left(X /{ }_{E}, \equiv\right)$ is a space of $E$-fuzzy points of $X$.

\section{Fuzzy Functions}

There are several definitions of a fuzzy function, see e.g. $[1,2,4,6]$. In $[1,2,4]$, a fuzzy function is considered as a special fuzzy relation. In [6], a fuzzy function is an ordinary mapping between two fuzzy spaces. In this section, a fuzzy function in the second sense will be considered as a mapping induced by the generalized extension principle, see subsection 2.1. We will investigate when a fuzzy function in the second sense is determined by a fuzzy function (relation) in the first sense.

In order to distinguish different notions, we will use the name " $(E-F)$-fuzzy function" for a fuzzy function which is a special fuzzy relation, and keep the simple name "fuzzy function" for that one which is a mapping between two fuzzy spaces. 


\section{1. $(E-F)$-fuzzy function}

Below, we recall the notion of a fuzzy function (see $[2,4])$ which will be further referred to as an $(E-F)$ fuzzy function.

\section{Definition 3}

Let $E, F$ be respective fuzzy equivalences on $X$ and $Y$. An $(E-F)$-fuzzy function is a binary fuzzy relation $\rho$ on $X \times Y$ such that for all $x, x^{\prime} \in X$, $y, y^{\prime} \in Y$ the following axioms hold true:

F.1 $\rho(x, y) * E\left(x, x^{\prime}\right) \leq \rho\left(x^{\prime}, y\right)$,

F.2 $\rho(x, y) * F\left(y, y^{\prime}\right) \leq \rho\left(x, y^{\prime}\right)$,

F.3 $\rho(x, y) * \rho\left(x, y^{\prime}\right) \leq F\left(y, y^{\prime}\right)$,

An $(E-F)$-fuzzy function is called perfect [4] if

F.4 for all $x \in X$, there exists $y \in Y$, such that $\rho(x, y)=1$.

An $(E-F)$-fuzzy function is called (strong) surjective $[3]$ if

F.5 for all $y \in Y$, there exists $x \in X$, such that $\rho(x, y)=1$.

Actually, an $(E-F)$-fuzzy function is not explicitly connected with an ordinary mapping between universes $X$ and $Y$. It establishes a double extensional connection between the $E$-space of $X$ and the $F$-space of $Y$ (axioms F.1, F.2) which is weakly functional (axioms F.3). However, if for all $x \in X$, $\rho(x, \cdot)$ is a normal fuzzy set (i.e $\rho$ is perfect) then there exists an ordinary function $g: X \longrightarrow Y$ such that for all $y \in Y, \rho(x, y)=F(g(x), y)$ (see [4]). This means that any point $y \in Y$ can be considered as a fuzzy value of $\rho$ at $x \in X$ with the degree equal to fuzzy equivalence $F$ between $y$ and $g(x)$.

In our investigation, we will consider an $(E-F)$ fuzzy function $\rho$ as a point-to-(fuzzy set) mapping on $X$ such that for all $x \in X$, the value $\rho(x, \cdot)$ is a fuzzy set on $Y$. Moreover, we will consider the case where $\rho$ is a surjective $(E-F)$-fuzzy function, so that there is no evident relation between an arbitrary point $x \in X$ and the kernel of $\rho(x, \cdot)$. In this case, we will show that a fuzzy value of $\rho$ at an arbitrary point $x \in X$ has more complicated meaning than that expressed above, see (17) and Remark 1 below. Nevertheless, we will prove (Theorem 2 below) that under more rigorous assumptions on the fuzzy equivalence $F$, there exist a subset $X^{\prime} \subseteq X$ and an ordinary function $g: X^{\prime} \longrightarrow Y$ such that for all $x^{\prime} \in X^{\prime}, y \in Y, \rho\left(x^{\prime}, y\right)=F\left(g\left(x^{\prime}\right), y\right)$. Additionally, we will propose an analytic representation of $\rho$ and use $\rho$ in the generalized extension principle.

\subsection{Fuzzy function as a mapping}

We will define a fuzzy function (see [6]) as a special correspondence between two fuzzy spaces. Let us remind that fuzzy function is a mapping which respects certain equality relation.

\section{Definition 4}

Let $\mathcal{L}$ be a residuated lattice and $\left(L^{X},=\right),\left(L^{Y},=\right)$ fuzzy spaces on $X$ and $Y$ respectively.

- A map $f: L^{X} \longrightarrow L^{Y}$ is a fuzzy function if for every $A, B \in L^{X}$,

$$
A=B \text { implies } f(A)=f(B) .
$$

The example of a fuzzy function is given below.

\section{Example 1}

\section{Fuzzy function determined by fuzzy relation}

Let $\mathcal{L}$ be a complete residuated lattice, $\left(L^{X},=\right)$, $\left(L^{Y},=\right)$ be fuzzy spaces on $X$ and $Y$ respectively, $R \in L^{X \times Y}$ a fuzzy relation. For every $A \in L^{X}$, we define a o-composition of $A$ and $R$ by

$$
(A \circ R)(y)=\bigvee_{x \in X}(A(x) * R(x, y)) .
$$

The o-composition determines a respective fuzzy set $A \circ R$ on $Y$. The corresponding mapping $f_{\circ R}: A \mapsto$ $A \circ R$ is a fuzzy function determined by $R$ on the whole fuzzy space $L^{X}$. It is easy to see that (6) is the discussed above generalization of the extension principle given by (2).

\section{Fuzzy Function Determined by an $(E-F)$-Fuzzy Function}

Our purpose is to investigate properties and relationship between an $(E-F)$-fuzzy function and determined by it fuzzy function (see Example 1 and expression (6).

\subsection{Core function of an $(E-F)$-fuzzy function}

In this section, we will show that each surjective $(E-F)$-fuzzy function $\rho$ on $X \times Y$ determines a respective ordinary core function $g: X^{\prime} \longrightarrow Y$, where $X^{\prime} \subseteq X$, such that at any $x^{\prime} \in X^{\prime}$, the value $\rho\left(x^{\prime}, \cdot\right)$ is equal to the $F$-fuzzy point $F_{g\left(x^{\prime}\right)}$. Moreover, in the below given theorems we will prove that at any $x^{\prime} \in X^{\prime}$, the value $\rho\left(x^{\prime}, \cdot\right)$ is equal to the value of the fuzzy function $f_{\circ \rho}$ at the respective $E^{\prime}$-fuzzy point $E_{x^{\prime}}^{\prime}$

\section{Theorem 1}

Let fuzzy relations $E$ on $X$ and $F$ on $Y$ be fuzzy equivalences and moreover, $F$ be a fuzzy equality. Let fuzzy relation $\rho$ on $X \times Y$ be a surjective $(E-F)$ fuzzy function, and $x_{y} \in X$ be such that $\rho\left(x_{y}, y\right)=$ $1, y \in Y$. Put $X^{\prime}=\left\{x_{y} \mid x_{y} \in X, y \in Y\right\}$. Then there exists the coarsest fuzzy equivalence $E^{\prime}$ on $X$ such that

(i) $E \leq E^{\prime}$ and $\rho$ is an $\left(E^{\prime}-F\right)$-fuzzy function,

(ii) for all $y, y^{\prime} \in Y$,

$$
E^{\prime}\left(x_{y}, x_{y^{\prime}}\right)=F\left(y, y^{\prime}\right)
$$


(iii) the mapping $g: X^{\prime} \longrightarrow Y$ such that $g\left(x_{y}\right)=y$ is functional and extensional with respect to $E^{\prime}$ and $F$, i.e. for all $x, t \in X^{\prime}$,

$$
E(x, t) \leq F(g(x), g(t)) .
$$

PROOF: Let all assumptions of the theorem be fulfilled. According to F.3, for all $y, y^{\prime} \in Y$,

$$
\bigvee_{x \in X} \rho(x, y) * \rho\left(x, y^{\prime}\right) \leq F\left(y, y^{\prime}\right)
$$

Similarly, by $\mathbf{F . 2}$, for all $y, y^{\prime} \in Y$,

$$
F\left(y, y^{\prime}\right) \leq \bigwedge_{x \in X}\left(\rho(x, y) \leftrightarrow \rho\left(x, y^{\prime}\right)\right)
$$

Thus,

$$
\bigvee_{x \in X} \rho(x, y) * \rho\left(x, y^{\prime}\right) \leq \bigwedge_{x \in X}\left(\rho(x, y) \leftrightarrow \rho\left(x, y^{\prime}\right)\right)
$$

Let $\left\{A_{y} \mid A_{y}(x)=\rho(x, y), y \in Y\right\}$ be a class of fuzzy sets on $X$. By the assumption, fuzzy sets $A_{y}$ are normal. By the inequality above, for all $y, y^{\prime} \in Y$,

$$
\bigvee_{x \in X} A_{y}(x) * A_{y^{\prime}}(x) \leq \bigwedge_{x \in X}\left(A_{y}(x) \leftrightarrow A_{y^{\prime}}(x)\right)
$$

It is known (see [2]) that normality of $A_{y}$ together with the inequality (11) guarantee that the following fuzzy relation

$$
E^{\prime}\left(x, x^{\prime}\right)=\bigwedge_{y \in Y}\left(A_{y}(x) \leftrightarrow A_{y}\left(x^{\prime}\right)\right),
$$

is a fuzzy equivalence on $X$. Let us verify that $E^{\prime}$ fulfils statements (i) - (iii).

(i) It has been proven in [2] that the fuzzy equivalence $E^{\prime}$ has fuzzy sets $A_{y}$ as $E^{\prime}$-equivalence classes so that for all $y \in Y$,

$$
A_{y}(x)=E^{\prime}\left(x, x_{y}\right), \quad x \in X .
$$

Moreover by (12), $E^{\prime}$ is the coarsest fuzzy equivalence relation on $X$ which fulfils $\mathbf{F . 1}$, so that

$$
E\left(x, x^{\prime}\right) \leq E^{\prime}\left(x, x^{\prime}\right), \quad x, x^{\prime} \in X,
$$

and $\rho$ is an $\left(E^{\prime}-F\right)$-fuzzy function.

(ii) The proof of (7) will be obtained by proving that for all $y, y^{\prime} \in Y$,

$$
\begin{aligned}
\bigvee_{x \in X} A_{y}(x) * A_{y^{\prime}}(x) & =\bigwedge_{x \in X}\left(A_{y}(x) \leftrightarrow A_{y^{\prime}}(x)\right)= \\
& =E^{\prime}\left(x_{y}, x_{y^{\prime}}\right),
\end{aligned}
$$

and

$$
\begin{aligned}
\bigvee_{x \in X} A_{y}(x) * A_{y^{\prime}}(x) & =\bigwedge_{x \in X}\left(A_{y}(x) \leftrightarrow A_{y^{\prime}}(x)\right)= \\
& =F\left(y, y^{\prime}\right),
\end{aligned}
$$

hold true.

The proof of (14) is split into the following four true inequalities:
(1) $\bigvee_{x \in X} A_{y}(x) * A_{y^{\prime}}(x)=\bigvee_{x \in X} E^{\prime}\left(x_{y}, x\right) *$ $E^{\prime}\left(x_{y^{\prime}}, x\right) \leq E^{\prime}\left(x_{y}, x_{y^{\prime}}\right)$

(2) $\bigvee_{x \in X} A_{y}(x) * A_{y^{\prime}}(x)=\bigvee_{x \in X} E^{\prime}\left(x_{y}, x\right) *$ $E^{\prime}\left(x_{y^{\prime}}, x\right) \geq E^{\prime}\left(x_{y}, x_{y^{\prime}}\right) * E^{\prime}\left(x_{y^{\prime}}, x_{y^{\prime}}\right)=$ $E^{\prime}\left(x_{y}, x_{y^{\prime}}\right)$

(3) $\bigwedge_{x \in X}\left(A_{y}(x) \quad \leftrightarrow \quad A_{y^{\prime}}(x)\right) \quad=$ $\bigwedge_{x \in X}\left(E^{\prime}\left(x_{y}, x\right) \quad \leftrightarrow \quad E^{\prime}\left(x_{y^{\prime}}, x\right)\right) \quad \geq$ $E^{\prime}\left(x_{y}, x_{y^{\prime}}\right)$,

(4) $\bigwedge_{x \in X}\left(A_{y}(x) \quad \leftrightarrow \quad A_{y^{\prime}}(x)\right) \quad=$ $\bigwedge_{x \in X}\left(E^{\prime}\left(x_{y}, x\right) \quad \leftrightarrow \quad E^{\prime}\left(x_{y^{\prime}}, x\right)\right) \leq$ $E^{\prime}\left(x_{y}, x_{y^{\prime}}\right) \leftrightarrow E^{\prime}\left(x_{y^{\prime}}, x_{y^{\prime}}\right)=E^{\prime}\left(x_{y^{\prime}}, x_{y^{\prime}}\right)$.

The proof of (15) is based on (9), (10) and the equality $A_{y}(x)=\rho(x, y)$. Indeed by $(9)$,

$$
\bigvee_{x \in X} A_{y}(x) * A_{y^{\prime}}(x) \leq F\left(y, y^{\prime}\right)
$$

and by (10),

$$
F\left(y, y^{\prime}\right) \leq \bigwedge_{x \in X}\left(A_{y}(x) \leftrightarrow A_{y^{\prime}}(x)\right) .
$$

By (14), $\bigvee_{x \in X} A_{y}(x) \quad * \quad A_{y^{\prime}}(x)=$ $\bigwedge_{x \in X}\left(A_{y}(x) \leftrightarrow A_{y^{\prime}}(x)\right)$, and thus (15) holds true. Finally by (14) and (15), we easily have (7).

(iii) Let $X^{\prime}=\left\{x_{y} \mid x_{y} \in X, y \in Y\right\}$. By (7) and the assumption that $F$ is a fuzzy equality on $Y$, the following statement holds: for all $y, y^{\prime} \in Y$, $y \neq y^{\prime}$ if and only if $x_{y} \neq x_{y^{\prime}}$. Therefore, the assignment $g: x_{y} \mapsto y$ defines a function on $X^{\prime}$. Extensionality of $g$ follows from (7) too. Indeed, for all $y, y^{\prime} \in Y$,

$$
E^{\prime}\left(x_{y}, x_{y^{\prime}}\right)=F\left(y, y^{\prime}\right)=F\left(g\left(x_{y}\right), g\left(x_{y^{\prime}}\right)\right) .
$$

Let the assumptions of Theorem 1 be fulfilled and $E^{\prime}$ be the fuzzy equivalence given by (12). Let us consider $E^{\prime}$-fuzzy points of $X$ (denoted by $E_{t}^{\prime}$ each) and $F$-fuzzy points of $Y$ (denoted by $F_{y}$ each) where $t \in X^{\prime}, y \in Y$. The respective sets of fuzzy points $\left\{E_{t}^{\prime} \mid t \in X^{\prime}\right\}$ and $\left\{F_{y} \mid y \in Y\right\}$ establish $*$-semipartitions of $X$ and $Y$. Moreover, the set $\left\{F_{y} \mid y \in\right.$ $Y\}$ is actually a *-partition of $Y$ (see[10]) in the sense that the set of kernels $\{y \mid t \in Y\}$ is equal to $Y$.

\subsection{Relationship between fuzzy function and $(E-F)$-fuzzy function}

The theorem below shows that two fuzzy functions, namely: an $(E-F)$-fuzzy function and a fuzzy function given by (6), coincide at respective "points" if they are determined by the same fuzzy relation $\rho$. Moreover, at common "points" both of them are determined by the same ordinary function which is a core function of the $(E-F)$-fuzzy function $\rho$ (see expression (18) below). 


\section{Theorem 2}

Let fuzzy relations $E, E^{\prime}, F, \rho$ and function $g$ : $X^{\prime} \longrightarrow Y$ where $X^{\prime}=\left\{x_{y} \mid \rho\left(x_{y}, y\right)=1, x_{y} \in\right.$ $X, y \in Y\}$ fulfil assumptions and conclusions of Theorem 1. Let moreover, $f_{\circ \rho}: L^{X} \longrightarrow L^{Y}$ be a fuzzy function determined by $\rho$ in accordance with (6), i.e. for all $A \in L^{X}$,

$$
f_{\circ \rho}(A)(y)=(A \circ \rho)(y)=\bigvee_{x \in X}(A(x) * \rho(x, y)) .
$$

\section{Then}

(i) for all $x \in X, y \in Y$,

$$
\rho(x, y)=\bigvee_{x^{\prime} \in X^{\prime}}\left(E_{x^{\prime}}^{\prime}(x) * F_{g\left(x^{\prime}\right)}(y)\right),
$$

(ii) for all $t \in X^{\prime}, y \in Y$,

$$
\rho(t, y)=f_{\circ \rho}\left(E_{t}^{\prime}\right)(y)=\left(E_{t}^{\prime} \circ \rho\right)(y)=F_{g(t)}(y) .
$$

\section{PROOF:}

(i) Let us choose $x \in X, y \in Y$. By (13), the lefthand side of (17) is equal to

$$
\rho(x, y)=A_{y}(x)=E^{\prime}\left(x, x_{y}\right) .
$$

By (7), (16) and the choice of $X^{\prime}$ and $g$, the right-hand side of (17) is equal to

$$
\begin{aligned}
& \bigvee_{x^{\prime} \in X^{\prime}}\left(E^{\prime}\left(x^{\prime}, x\right) * F\left(g\left(x^{\prime}\right), y\right)\right)= \\
& \bigvee_{y^{\prime} \in Y}\left(E^{\prime}\left(x_{y^{\prime}}, x\right) * F\left(g\left(x_{y^{\prime}}\right), y\right)\right)= \\
& \bigvee_{y^{\prime} \in Y}\left(E^{\prime}\left(x_{y^{\prime}}, x\right) * F\left(g\left(x_{y^{\prime}}\right), g\left(x_{y}\right)\right)\right)= \\
& \bigvee_{y^{\prime} \in Y}\left(E^{\prime}\left(x_{y^{\prime}}, x\right) * F\left(y^{\prime}, y\right)\right)= \\
& \bigvee_{y^{\prime} \in Y}\left(E^{\prime}\left(x_{y^{\prime}}, x\right) * E^{\prime}\left(x_{y^{\prime}}, x_{y}\right)=\right. \\
& \bigvee_{x_{y^{\prime}} \in X^{\prime}}\left(E^{\prime}\left(x_{y^{\prime}}, x\right) * E^{\prime}\left(x_{y^{\prime}}, x_{y}\right)\right.
\end{aligned}
$$

where an arbitrary element $x^{\prime} \in X^{\prime}$ has been identified with $x_{y^{\prime}}$.

Thus, to prove (17) it is enough to prove that

$$
E^{\prime}\left(x, x_{y}\right)=\bigvee_{x_{y^{\prime}} \in X^{\prime}}\left(E^{\prime}\left(x_{y^{\prime}}, x\right) * E^{\prime}\left(x_{y^{\prime}}, x_{y}\right)\right.
$$

By transitivity of $E^{\prime}$,

$$
\bigvee_{x_{y^{\prime}} \in X^{\prime}}\left(E^{\prime}\left(x_{y^{\prime}}, x\right) * E^{\prime}\left(x_{y^{\prime}}, x_{y}\right) \leq E^{\prime}\left(x, x_{y}\right)\right.
$$

On the other hand,

$$
\begin{gathered}
\bigvee_{x_{y^{\prime}} \in X^{\prime}}\left(E^{\prime}\left(x_{y^{\prime}}, x\right) * E^{\prime}\left(x_{y^{\prime}}, x_{y}\right) \geq\right. \\
E^{\prime}\left(x_{y}, x\right) * E^{\prime}\left(x_{y}, x_{y}\right)=E^{\prime}\left(x, x_{y}\right) .
\end{gathered}
$$

Both inequalities together with (19) prove (17). (ii) Let us consider the following system of fuzzy relation equations:

$$
E_{t}^{\prime} \circ R=F_{g(t)}, \quad t \in X^{\prime},
$$

where $R$ is an unknown fuzzy relation on $X \times$ $Y$. According to the respective "easy-to-checkcriterion" (see [12]), and by the facts that fuzzy points of $\left(X, E^{\prime}\right)$ and $(Y, F)$ establish *-semipartitions of $X$ and $Y$ and these semi-partitions are connected by the extensional (in the sense of (8)) function $g$, the fuzzy relation $\bigvee_{t \in X^{\prime}}\left(E_{t}^{\prime} *\right.$ $\left.F_{g(t)}\right)$ on $X \times Y$ given by

$$
\begin{aligned}
& \left(\bigvee_{t \in X^{\prime}} E_{t}^{\prime} * F_{g(t)}\right)(x, y)= \\
& \bigvee_{t \in X^{\prime}}\left(E^{\prime}(t, x) * F(g(t), y)\right), \quad x \in X, y \in Y,
\end{aligned}
$$

is one of solutions of (20). By (17), the above fuzzy relation is equal to $\rho$. Therefore, $\rho$ fulfils (20), i.e.

$$
E_{t}^{\prime} \circ \rho=F_{g(t)}, \quad t \in X^{\prime} .
$$

By (6),

$$
f_{\circ \rho}\left(E_{t}^{\prime}\right)=E_{t}^{\prime} \circ \rho=F_{g(t)}, \quad t \in X^{\prime},
$$

which is the right-hand side equality in (18). Finally, let us prove (18) by proving that for all $t \in X^{\prime}, y \in Y$,

$$
\rho(t, y)=F_{g(t)}
$$

Let us choose $t \in X^{\prime}$ and $y \in Y$. By (16), (17) and (20),

$$
\begin{aligned}
& \rho(t, y)=\bigvee_{x^{\prime} \in X^{\prime}}\left(E^{\prime}\left(x^{\prime}, t\right) * F\left(g\left(x^{\prime}\right), y\right)\right)= \\
& \bigvee_{x^{\prime} \in X^{\prime}}\left(E^{\prime}\left(x^{\prime}, t\right) * F\left(g\left(x^{\prime}\right), g\left(x_{y}\right)\right)\right)= \\
& \bigvee_{x^{\prime} \in X^{\prime}}\left(E^{\prime}\left(x^{\prime}, t\right) * E^{\prime}\left(x^{\prime}, x_{y}\right)\right)= \\
& E^{\prime}\left(t, x_{y}\right)=F\left(g(t), g\left(x_{y}\right)\right)=F(g(t), y)=F_{g(t)} .
\end{aligned}
$$

\section{Remark 1}

Let us analyze expressions (17) and (18). Both of them represent fuzzy values of an $(E-F)$-fuzzy function $\rho$ at points of $X$. If $x \notin X^{\prime}$, i.e. $x$ is not related with a certain point in $Y$, then by (17), the computation of $\rho(x, \cdot)$ involves all $E^{\prime}$-fuzzy points of $X^{\prime}$ and all respective $F$-fuzzy points of $Y$. This means that any point $y \in Y$ can be considered as a fuzzy value of $\rho$ at $x \in X$ with the degree equal to the greatest value of the product between fuzzy equivalence $E^{\prime}$ between $x$ and $x^{\prime}$ and fuzzy equivalence $F$ between $y$ and $g\left(x^{\prime}\right)$. 
On the other side, if $x \in X^{\prime}$ then the value $\rho(x, \cdot)$ does not require any computation, because by (18), it is equal to the $F$-fuzzy point $F_{g(x)}$. Moreover, if $x \in X^{\prime}$ then by (18), the same conclusion is valid for the value of the fuzzy function $f_{\circ \rho}$ at the $E^{\prime}$-fuzzy point $E_{x}^{\prime}$.

\section{3. (Fuzzy point)-to-(Fuzzy point) representation of fuzzy functions}

Our next goal is to find conditions that assure equality $X=X^{\prime}$. In this case, computation of $\rho(x, \cdot)$ at $x \in X$ and $f_{\circ \rho}\left(E_{x}^{\prime}\right)$ at the related to $x E^{\prime}$-fuzzy point $E_{x}^{\prime}$ can be done on the basis of (18).

\section{Theorem 3}

Let fuzzy relations $E$ on $X$ and $F$ on $Y$ be fuzzy equivalences and moreover, $F$ be a fuzzy equality. Let function $g: X \longrightarrow Y$ be surjective, i.e. $g(X)=$ $Y$, and fuzzy relation $\rho_{g}$ be given by

$\rho_{g}(x, y)=\bigvee_{x^{\prime} \in X}\left(E\left(x^{\prime}, x\right) * F\left(g\left(x^{\prime}\right), y\right)\right), x \in X, y \in Y$.

Then $\rho_{g}$ is an $(E-F)$-fuzzy function if and only if function $g$ is extensional with respect to $E$ and $F$, i.e $g$ fulfils (8).

\section{PROOF:}

(i) Let all the assumptions of the theorem be fulfilled and moreover, function $g$ be extensional with respect to $E$ and $F$. The proof that the fuzzy relation $\rho_{g}$ is an $(E-F)$-fuzzy function is given in [2].

(ii) Let all the assumptions of the Theorem be fulfilled, and the fuzzy relation $\rho_{g}$, given by $(24)$, be an $(E-F)$-fuzzy function. We will prove that the function $g$ is extensional with respect to $E$ and $F$.

By the assumption, the function $g: X \longrightarrow Y$ is surjective i.e., for each $y \in Y$, there exists $x_{y} \in X$ such that $g\left(x_{y}\right)=y$. Therefore, by (24),

$$
\begin{gathered}
\rho_{g}\left(x_{y}, y\right)=\bigvee_{x^{\prime} \in X}\left(E\left(x^{\prime}, x_{y}\right) * F\left(g\left(x^{\prime}\right), g\left(x_{y}\right)\right)\right) \geq \\
E\left(x_{y}, x_{y}\right) * F\left(g\left(x_{y}\right), g\left(x_{y}\right)\right)=1 .
\end{gathered}
$$

Moreover, the set $\left\{x_{y} \mid y \in Y\right\}$ is equal to $X$, because the latter is the domain of $g$. By the proof of Theorem 1 , the fuzzy relation

$$
E^{\prime}\left(x, x^{\prime}\right)=\bigwedge_{y \in Y}\left(\rho(x, y) \leftrightarrow \rho\left(x^{\prime}, y\right)\right)
$$

is a fuzzy equivalence on $X$ such that for all $x, x^{\prime} \in X$,

$$
E\left(x, x^{\prime}\right) \leq E^{\prime}\left(x, x^{\prime}\right) .
$$

It has been proved in Theorem 1 that for all $x, x^{\prime} \in X, E^{\prime}\left(x, x^{\prime}\right)=F\left(g(x), g\left(x^{\prime}\right)\right)$. Therefore, $E\left(x, x^{\prime}\right) \leq F\left(g(x), g\left(x^{\prime}\right)\right)$ and thus, $g$ is extensional with respect to $E$ and $F$.
The corollary below shows that under the conditions of Theorem 3 , values $\rho_{g}(x, \cdot)$ and $f_{\circ \rho_{g}}\left(E_{x}\right)$ at $x \in X$, coincide with $F_{g(x)}$. Therefore, both fuzzy functions coincide at related to each other points.

\section{Corollary 1}

Let fuzzy relations $E$ on $X$ and $F$ on $Y$ be fuzzy equivalences and moreover, $F$ be a fuzzy equality. Let function $g: X \longrightarrow Y$ be surjective and extensional with respect to $E$ and $F$. Let moreover, fuzzy relation $\rho_{g}$ be given by (24). Then for all $x \in X, y \in Y$,

$$
\rho_{g}(x, y)=f_{\circ \rho_{g}}\left(E_{x}^{\prime}\right)=E_{x}^{\prime} \circ \rho_{g}=F_{g(x)} .
$$

PROOF: By Theorem 3, the fuzzy relation $\rho_{g}$ is an $(E-F)$-fuzzy function. Then equation (25) follows from Theorem 2 and equation (18).

\section{Remark 2}

Theorem 3 and its Corollary give two analytic representations of an $(E-F)$-fuzzy function $\rho_{g}$ which is connected with an ordinary core function $g$. By (24), $\rho_{g}$ is characterized as a fuzzy relation which connects each $E$-fuzzy point $E_{x}$ with the respective $F$-fuzzy point $F_{g(x)}$. In this case, we can say that (24) is (fuzzy point)-to-(fuzzy point) representation of $\rho_{g}$. The second representation is given by (25), and it identifies the fuzzy set value of $\rho_{g}$ at point $x \in X$ with the $F$-fuzzy point $F_{g(x)}$.

Similarly to Theorem 3 , the below given Theorem 4 contains necessary and sufficient conditions guaranteeing that a fuzzy function is determined by a fuzzy relation which has a (fuzzy point)-to-(fuzzy point) representation. Again we will see that a core function is involved. Theorem 4 has been proven in [12] and thus we will not repeat the proof.

\section{Theorem 4}

Let $E$ on $X$ be a fuzzy equivalence, $F$ on $Y$ be a fuzzy equality, and fuzzy function $f: X / E \longrightarrow$ $Y /{ }_{F}$ establish a correspondence between all $E$-fuzzy points of $X$ and $F$-fuzzy points of $Y$. Let moreover, function $g: X \longrightarrow Y$ be such that for all $x^{\prime} \in X$, $g\left(x^{\prime}\right) \in \operatorname{Ker}\left(f\left(E_{x^{\prime}}\right)\right)$. Then $f$ is determined by the following fuzzy relation $R_{g}$ on $X \times Y$ :

$$
R_{g}(x, y)=\bigvee_{x^{\prime} \in X}\left(E\left(x^{\prime}, x\right) * F\left(g\left(x^{\prime}\right), y\right)\right)
$$

i.e., for all $x \in X$,

$$
f_{\circ R_{g}}\left(E_{x}\right)=E_{x} \circ R_{g}=F_{g(x)},
$$

if and only if $g$ is extensional with respect to $E$ and $F$. 


\section{Conclusion}

Two approaches to the notion of fuzzy function have been investigated: the first one identifies a fuzzy function with a special fuzzy relation (we call it an $(E-F)$-fuzzy function), and the second one defines a fuzzy function as an ordinary mapping between fuzzy spaces. We combined both approaches and investigated properties of a fuzzy function determined by an $(E-F)$-fuzzy function. We found conditions that assure that dependent values of thus related fuzzy functions coincide. These conditions are based on the existence of a core function of the respective $(E-F)$-fuzzy function. Moreover, we investigated properties and relationship of related fuzzy functions in the case when they are "fuzzified" versions of an ordinary core function.

\section{References}

[1] P. Hájek. Metamathematics of Fuzzy Logic. Kluwer, Dordrecht, 1998.

[2] F. Klawonn. Fuzzy points, fuzzy relations and fuzzy functions. In V. Novák and I. Perfilieva, editors, Discovering the World with Fuzzy Logic, pages 431-453. Springer, Berlin, 2000.

[3] M. Demirci. Fuzzy functions and their fundamental properties. Fuzzy Sets and Systems, 106:239 - 246, 1999.

[4] M. Demirci. Fundamentals of m-vague algebra and $\mathrm{m}$-vague arithmetic operations. Int. Journ. Uncertainty, Fuzziness and KnowledgeBased Systems, 10:25-75, 2002.

[5] L. A. Zadeh. The concept of a linguistic variable and its application to approximate reasoning I, II, III. Information Sciences, 8-9:199257, 301-357, 43-80, 1975.

[6] I. Perfilieva. Fuzzy function as an approximate solution to a system of fuzzy relation equations. Fuzzy Sets and Systems, 147:363-383, 2004.

[7] V. Novák. Fuzzy Sets and Their Applications. Adam Hilger, Bristol, 1989.

[8] F. Klawonn and J. L. Castro. Similarity in fuzzy reasoning. Mathware Soft Comput., pages 197-228, 1995.

[9] U. Höhle. Many-valued equalities, singletons and fuzzy partitions. Soft Computing, 2:134140, 1998.

[10] B. De Baets and R. Mesiar. T-partitions. Fuzzy Sets Systems, 97:211-223, 1998.

[11] I. Perfilieva. Finitary solvability conditions for systems of fuzzy relation equations. Information Sciences, 2011.

[12] I. Perfilieva. Solvability of a system of fuzzy relation equations: Easy to check conditions. Neural Network World, 13:571-580, 2003. 